
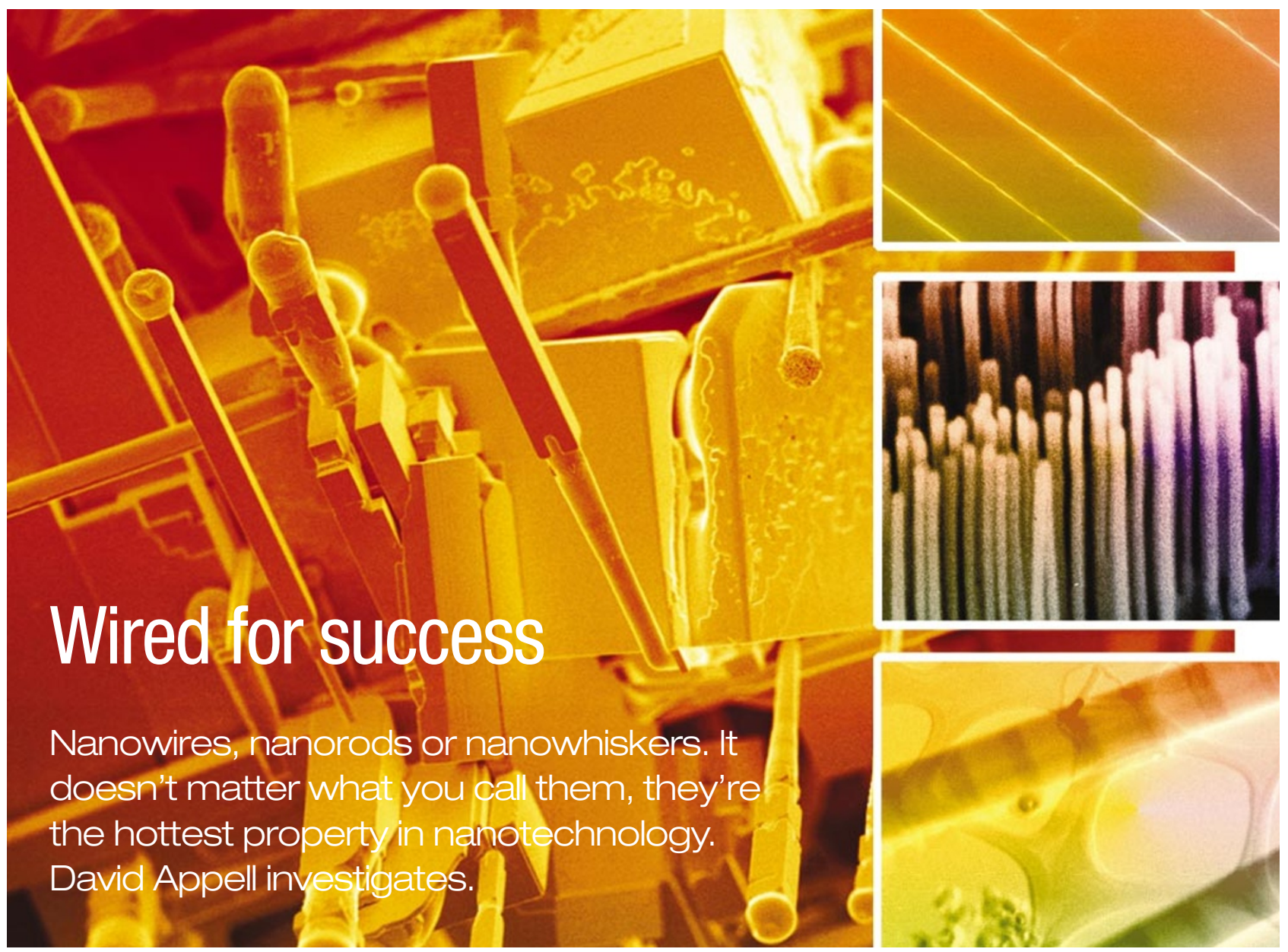

$\mathrm{n}$ the much-hyped world of nanotechnology, the carbon nanotube has hogged the limelight. These tiny hollow tubes, which resemble rolls of wire mesh, have already been fashioned into miniature tweezers that could one day be used to pick up and move individual cells. They might also form the basis of nanometre-sized computer circuits, according to advocates ${ }^{1}$. And some NASA researchers have even suggested that the tubes could be used to build space elevators to ferry people and satellites into orbit.

But away from the spotlight, nanotube researchers have hit problems. Potential computing applications have generated the most excitement, but some of the tubes' crucial electronic properties, which depend on precise orientation of the carbon mesh, are hard to control. The difficulties may not be insurmountable, but they have persuaded some scientists to turn their attention elsewhere. Over the past few years, alternative nanoscale structures have attracted attention. One in particular - called either the nanowire, nanorod or nanowhisker, depending on which researcher you ask has gained an impressive following.

These nanometre-diameter wires, which can be grown from a variety of different materials and can reach tens of micrometres in length, have one major advantage - unlike nanotubes, their chemistry is relatively easy to tailor. By growing nanowires from different semiconductors - materials whose conductivity lies somewhere between those of a conductor and an insulator — some researchers have already used them to build transistors and diodes. Others have constructed lasers and biological sensors. "There are a lot of unknowns to be discovered," says Charles Lieber, a Harvard University chemist who has abandoned much of his work with nanotubes to focus on nanowires. "If you're creative, there are very different things to do with these materials."

\section{The chips are down}

Both nanotubes and nanowires are attracting attention because of a looming crisis in computing. Traditional electronic circuits are built by etching individual components into silicon wafers. Advances in this technique have ensured that computer chips have steadily shrunk in size over the past few decades. But the circuits can only become so small - current fabrication methods will fail well before the size of electronic components reaches atomic dimensions, probably within the next 20 years.
Ahead by a whisker: semiconductor nanowires (main picture) are relatively easy to make. They can be aligned in channels (inset, top), grown in arrays (inset, middle) or produced from alternating materials as supperlattices (bottom).

Electronic circuits built from nanowires or nanotubes are one way in which the miniaturization trend could be continued. The wires on today's cutting-edge computer chips are a few hundred nanometres thick nanowire or nanotube alternatives could be a least an order of magnitude thinner. Other more complex components of electronic circuits, such as switches and light-emitting diodes, could also be built from nanowires or nanotubes.

Progress with nanotubes has been impressive since they were first discovered just over a decade ago $^{2}$. For example, transistors - electronic devices that can act as switches and can amplify current - have been built from nanotubes, and wired together to form logic gates, the building blocks of computers ${ }^{1}$. But to create these devices, researchers must carefully tweak the electronic properties of the nanotubes. This is not always easy to do, as these properties depend on the diameter and crystal structure of the tubes - parameters that are difficult 
to manipulate. "Carbon nanotubes have unique attributes," says Lieber. "But one has a difficult time controlling their metallic and semiconductor properties."

The composition of nanowires, by contrast, is relatively easy to control. The most popular method for producing them involves vapourliquid-solid (VLS) growth, first proposed in the 1960s but only coming into its own for nanowires in 1998, when Lieber's group combined it with other techniques ${ }^{3,4}$. To use VLS to make a silicon nanowire, for example, a laser is first used to vaporize the silicon together with a metal catalyst, typically iron or gold. As the vapour cools it forms nanosized liquid clusters containing both silicon and some iron. Silicon wires then slowly grow out from the cluster, as more silicon atoms condense.

If the nanowire is grown from a semiconductor, its electrical properties can be altered by adding small amounts of a second substance, called a dopant.

Adding boron to a silicon nanowire, for example, increases the number of 'holes' within the network of electrons available for conduction, whereas adding phosphorus creates additional free electrons ${ }^{5}$. James Ellenbogen, a nanowire researcher at MITRE, an engineering and computing firm in Bedford, Massachusetts, says that this ability to control the composition of nanowires is key. "One word distinguishes what semiconductor nanowires have that carbon nanotubes don't," he says. "Chemistry."

\section{Up the junction}

In conventional circuits, combinations of differently doped semiconductors are used to build electronic devices, and the same can be done using doped nanowires. Last year, for example, Harvard chemist Yi Cui, together with Lieber, created two differently doped silicon semiconductor nanowires and joined them together. The difference in the density of conducting electrons has a very useful effect on the junction where the wires meet: it behaves as a diode, allowing current to flow in only one direction across it ${ }^{6}$.

This January, Yu Huang, another member of Lieber's group, described how he formed a cross using two different nanowires - one made of gallium nitride, the other of silicon - to create a transistor ${ }^{7}$. Such transistors could be the basis of a nanowire memory unit. The Harvard team has also coated vari-

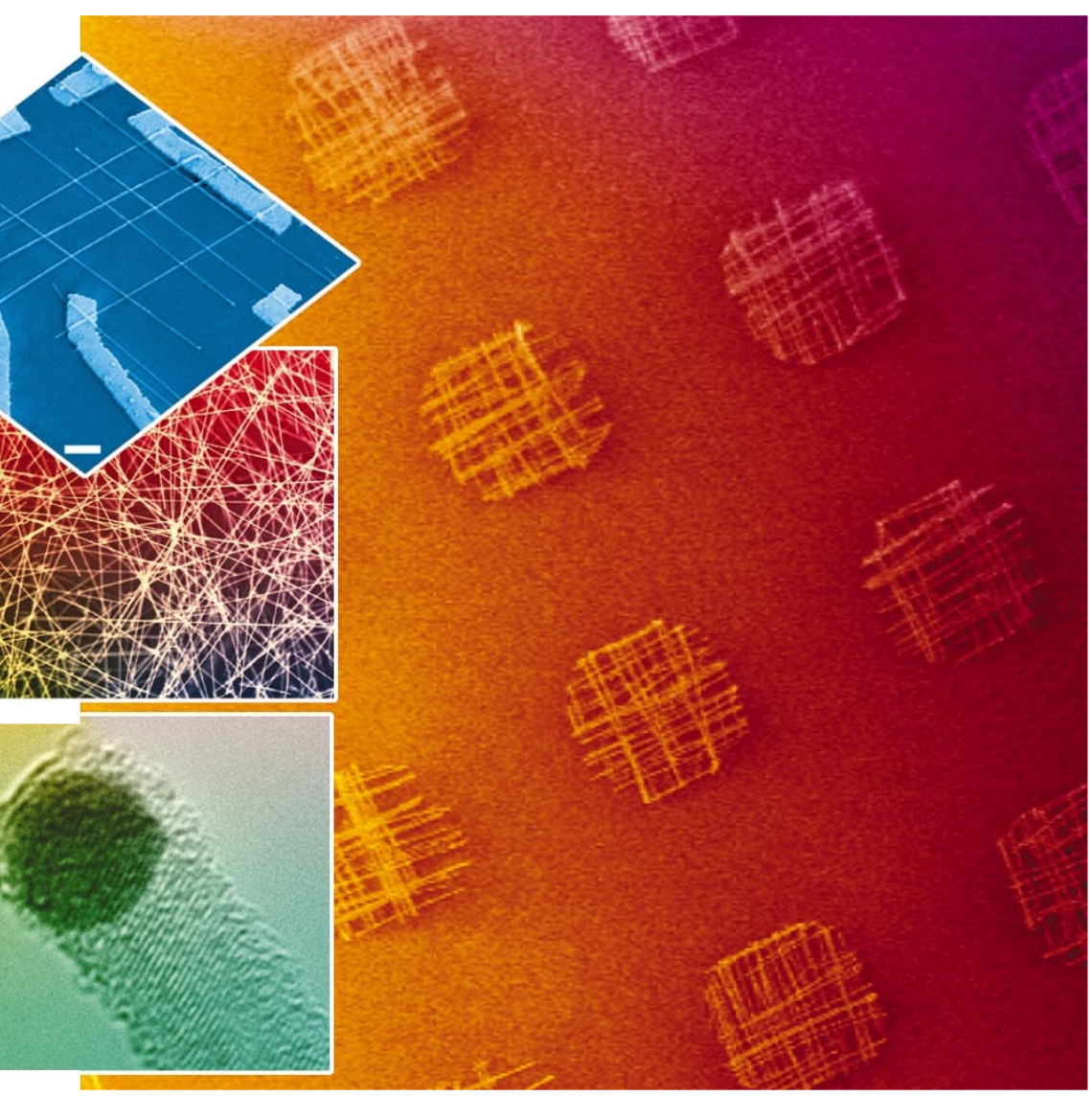

Memories are made of this: crossed nanowire arrays (main picture) and an experimental $4 \times 4$ array (inset, top) are being tested for use as computer memory. The nanowires (inset, middle) are readily grown on metal catalysts such as a gold nanoparticle (black spot, inset, bottom).

ous nanowires with charged molecules and shown that the charge affects the nanowires' ability to conduct electricity. By applying a voltage to change the charge, the researchers could switch the wire's conductance between two different states $^{8}$, in effect creating a nanowire memory that stores information in the form of the wire's conductance.

Speaking at the Nanotube 2002 conference, held this July in Boston, Lieber described how he had arranged four nanowires in the shape of a noughts and crosses (tic-tac-toe) grid. Each of the four junctions where two wires cross acts as a transistor. The conductance of the junction can be shifted between two states by applying a voltage across the two wires in the junction, allowing the system to act as a 4-bit memory.

Such a device could help the Molecular Electronics (Moletronics) programme run by the Defense Advanced Research Projects Agency, part of the US Department of Defense, to meet its goal. By September 2004, the Moletronics researchers hope to create 16 kilobits of self-assembled nanocomputer memory, possibly using nanowires, and to be able to interface the memory with macroscopic devices. Researchers at MITRE and other Moletronics-funded groups have begun designing and simulating architectures to scale up Lieber's array. They aim to create a system of $125 \times 125$ nanowires, about 5 micrometres long and only a few nanometres thick - if they succeed, this would come close to providing the 16 kilobits of memory.

Nanowire memories could also be far cheaper to run than existing ones. Conventional transistors need to be refreshed up to 1,000 times a second, or the charge on the transistor leaks away and the information is lost. But the charge on Lieber's nanowires remains in place for about 20 minutes $^{8}$. If such advantages could be scaled up, power demands for such devices could be cut by a factor of a million.

\section{Battle of the bands}

Since the start of this year, three groups Lieber's ${ }^{9}$ and those of Peidong Yang at the University of California, Berkeley ${ }^{10}$, and Lars Samuelson at Lund University in Sweden ${ }^{11}$ - have described how to build more complex functions into a single nanowire. By alternating between two vaporized materials during VLS, they have grown striped or 'superlattice' nanowires consisting of up to 21 alternating bands.

Each stripe contributes its own electronic 
properties to the nanowire, with some useful results. A nanowire consisting of alternating bands of differently doped indium phosphide, for example, glows when a current is passed through it and could form the basis of a nanowire light-emitting diode ${ }^{9}$. Yang has suggested that the thermal properties of some striped nanowires could make them suitable for building switches that respond to changes in temperature ${ }^{10}$. Moreover, the technique used to make superlatticed nanowires is cheap and efficient - millions can be created in just one hour at relatively low cost. "They have opened up a new world for us," says Lieber.

So can these individual components be assembled into a working device? The backers of the Moletronics programme clearly think so. But for practical applications, researchers will have to learn how to manipulate nanowires - a tougher challenge than one might expect. The random movement of electrons in nanowires creates areas of positive and negative charge, which causes two wires to cling together. "Some people would think it would be very hard to get nanowires to stick together," says Ellenbogen. "Actually it's very hard to pry them apart."

This sticky problem can be avoided by manipulating the wires in solution. Long organic molecules are added to the outside of the nanowires to act as a physical barrier. Yang's group has manipulated nanowires by filling a trough with a nanowire solution and increasing the pressure on the liquid. This causes the nanowires to condense out of solution and align themselves side by side ${ }^{12}$.

Crossed arrays can be formed by rotating the apparatus $90^{\circ}$ and repeating the process. By combining layers with different electrical properties, the junctions where the wires cross can be made to function as diodes or transistors, although all of the arrays built so far are proofs of principle rather than functioning devices.

\section{Blazing a trail}

In the meantime, other potential applications are being explored. Last year, for example, Yang's group created nanowires that emit laser light of an ultraviolet ${ }^{13}$ or deep blue colour. The team also showed that zinc oxide nanowires can be used to guide light, in much the same way as an optical fibre ${ }^{13}$. These advances lend hope to the idea that electronic circuits built from nanowires could eventually be integrated with optical systems, such as the fibres that carry voice and Internet data over long distances.

Nanowires might also form the basis of high-definition televisions. The electrons needed for the cathode-ray guns within television screens can be pulled from the tips of either nanowires or nanotubes, and the sharp ends of these structures allow lower-level electric fields to be used. Elec-
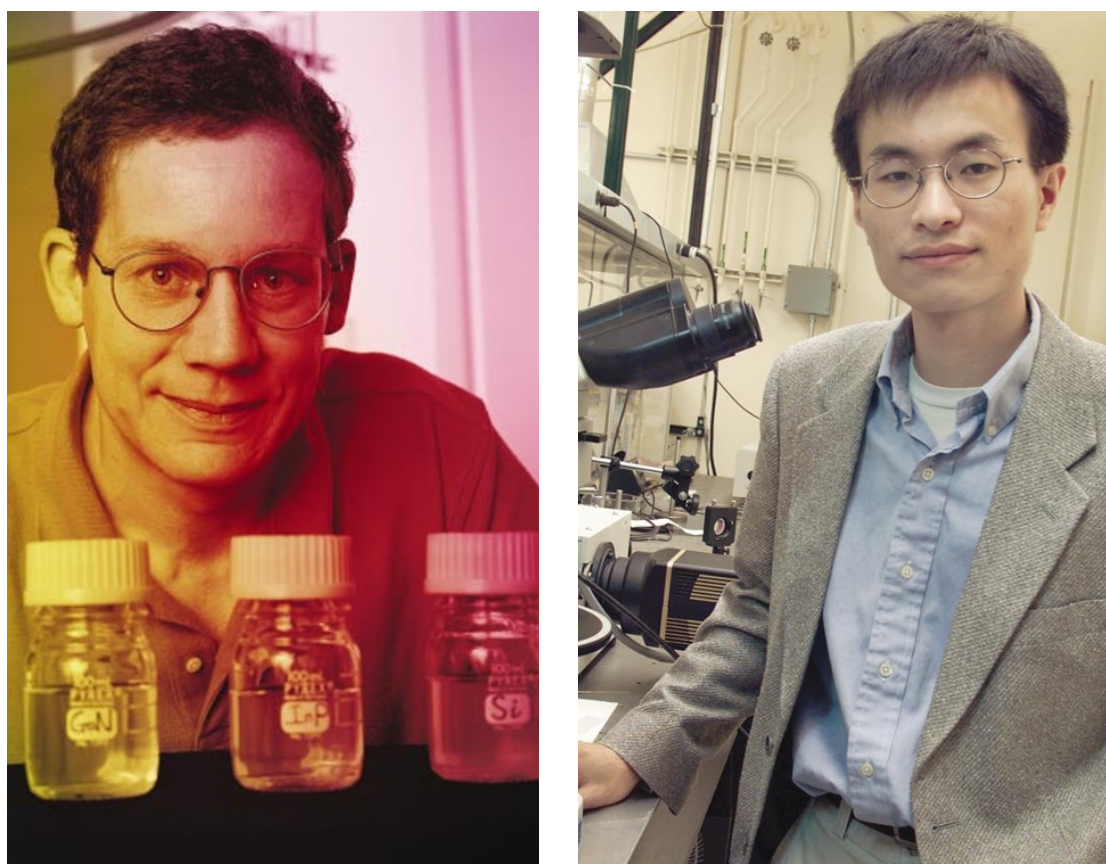

Keen edge: Charles Lieber (left) and Peidong Yang have been impressed by the versatility of nanowires.

tronics company Samsung has recently produced a prototype display using carbon nanotubes. Yang envisions a display that uses a single nanowire as one pixel, giving $10^{11}$ pixels per square centimetre - orders of magnitude beyond what can be achieved today.

New chemical and biological sensors could also be built from nanowires. Many molecules change the conductance of a nanowire when they bind to its surface. By monitoring this property, Lieber's group has built a nanowire sensor that can measure the $\mathrm{pH}$ of a solution ${ }^{14}$. And in unpublished work, the team has found that by placing antibodies along the wire, it can serve as a sensor for prostate-specific antigen (PSA), which marks the presence or recurrence of prostate cancer. The device can detect levels of PSA four times smaller than is now possible with blood tests that can take days, says Lieber.

The PSA sensor has been licensed to Nanosys of Palo Alto, California, a company set up by Lieber in 2001 that has already raised more than $\$ 15$ million in two rounds of funding. Such devices look promising, but whether Nanosys or another company will ever achieve nanotechnology's main goal to compete with conventional computers remains unclear.

Working nanowire memory units would be tiny and cheap to run, but they will not become part of everyday computers in the near future. Researchers still have to figure out how such delicate components can be connected to the relatively gigantic wires in conventional microchips. They will also have to show that such units can compete with traditional memory systems in terms of reli- ability - a tough challenge given the sums that have been invested in developing conventional computers.

Lieber is unsure when nanowires will replace traditional computers - if at all. "Can we actually ever exceed what's been done? I think that it's too early to say," he says. "There's a big jump between the promise and the real technology. It will come, but people need to be a little patient."

But with nanowires showing so much potential, both researchers and funding organizations should be happy with the pace. Applications have mushroomed in the few years since VLS techniques were introduced, and many new directions await exploration. At the very least, nanowires look set to share the nanotechnology spotlight over the next few years. "Some of the things that have happened have exceeded anybody's expectations," Ellenbogen says. "I think there are more surprises along the way."

David Appell is a freelance science writer in Gilford,

\section{New Hampshire.}

1. Ball, P. Nature 414, 142-144 (2001)

2. Iijima, S. Nature 354, 56-58 (1991).

3. Morales, A. M. \& Lieber, C. M. Science 279, 208-211 (1998).

4. Duan, X. \& Lieber, C. M. Adv. Mater. 12, 298-302 (2000).

5. Cui, Y., Duan, X., Hu, J. \& Lieber, C. M. J. Phys. Chem. B 104, 5213-5216 (2000).

6. Cui, Y. \& Lieber, C. M. Science 291, 851-853 (2001)

7. Huang, Y., Duan, X., Cui, Y. \& Lieber, C. M. Nano Lett. 2, 101-104 (2002).

8. Duan, X., Huang, Y. \& Lieber, C. M. Nano Lett. 2, 487-490 (2002)

9. Gudiksen, M. S., Lauhon, L. J., Wang, J., Smith, D. C. \& Lieber, C. M. Nature 415, 617-620 (2002).

10. Wu, Y., Fan, R. \& Yang, P. Nano Lett. 2, 83-86 (2002).

11. Björk, M. T. et al. Nano Lett. 2, 87-89 (2002).

12. Kim, F., Kwan, S., Akana, J. \& Yang, P. J. Am. Chem. Soc. 123, 4360-4361 (2001).

13. Huang, M. H. et al. Science 292, 1897-1899 (2001).

14. Cui, Y., Wei, Q., Park, H. \& Lieber, C. M. Science 293, 1289-1292 (2001). 